\title{
Bol E-Tümlenmiş Modüllere ve E-Yükseltilebilir Modüllere Torsiyon-Teorik Bir Yaklaşım
}

\author{
Esra Öztürk Sözen ${ }^{1 *}$ \\ ${ }^{1}$ Sinop Üniversitesi, Fen Edebiyat Fakültesi, Matematik Bölümü
}

Geliş / Received: 17/02/2020, Kabul / Accepted: 01/06/2020

\section{$\ddot{O} z$}

Bu çalışmada bol $\tau_{e}$-tümlenmiş ve $\tau_{e}$-yükseltilebilir modülleri tanımlıyoruz ve bu yapıların temel özelliklerini araştırıyoruz. Ayrıca $\tau_{e}$-tümlenmiş, bol $\tau_{e}$-tümlenmiş ve $\tau_{e}$-yükseltilebilir modüllerin arasındaki ikili ilişkileri belirtiyoruz.

Anahtar Kelimeler: $e$-küçük alt modül, bol $e$-tümlenmiş modül, $e$-yükseltilebilir modül, kalıtsal torsiyon teorisi.

\section{A Torsion-Theoretic Approximation to Amply $e$-Supplemented Modules and $\boldsymbol{e}$-Lifting Modules}

\begin{abstract}
In this study, we define amply $\tau_{e}$-supplemented and $\tau_{e}$-lifting modules and investigate main structures of them. Moreover we determine their relationship $\tau_{e}$-supplemented, amply $\tau_{e}$-supplemented and $\tau_{e}$-lifting modules with each other' düzenlemesi yapılabilir.
\end{abstract}

Keywords: $e$-small submodule, amply $e$-supplemented module, $e$-lifting module, hereditary torsion theory.

\section{Giriș}

Bu çalışmada $R, M$ ve $\tau$ notasyonları sırasıyla birimli halkaları, üniter $R$-modülleri ve kalitsal torsiyon teorisini belirtecektir. $M$ modülünün bir $X$ alt modülü için $X+Y=M$ koşulu yalnızca $Y=M \quad$ olduğunda sağlanıyorsa, $X$ alt modülüne $\mathrm{M}$ nin küçük alt modülü denir ve " $X \ll M^{\prime \prime}$ ile gösterilir. Diğer $\operatorname{taraftan} X \leq M$ alt modülünün $M$ nin sıfır alt modülünden farklı tüm öz alt modülleri ile arakesiti sıfırdan farklı oluyorsa, $X$ alt modülüne $M$ nin büyük alt modülüdür denir. Tümlenmiş modül, projektif örtü, ve dolayısıyla yarı mükemmel modüllerin tanımlanmasında kilit rol üstlenen küçük alt modüller bu yönüyle modül ve halka teorisinin odağında kalmayı hak etmektedir. Ayrica 2011 yılında Zhou ve Zang tarafindan, küçük ve büyük alt modüllerden esinlenerek tanımlanan, $e$-küçük alt modül kavramı da bu alanda güncel pek çok çalışmanın ortaya konmasına olanak sağlamıştır (Quynh ve Tin, 2013; Koşar ve ark., 2015). Bu sayede $e$ tümlenmiş modül, $e$-yükseltilebilir modül gibi kavramlar ve bir takım genelleştirmeleri literatüre kazandırılmıştır.

Bir modülün küçük ve büyük alt modüllerinin torsiyon teorik versiyonları ilk olarak 1998 yılında Bland tarafından verilmiştir. 2007 
yılında Charalambides ve Clark CS modülleri torsiyon teorisine göre irdelemiştir. 2004 yılında ise Harmancı ve Koşan' in tümlenmiş modüllerin kalitsal torsiyon teorisine göre varyasyonunu sunan çalışmaları yayımlanmıştır.

Bu çalışmada da $e$-tümlenmiş modüllerin, bol $e$-tümlenmiş modül ve $e$-yükseltilebilir modül gibi iki özel genelleştirmesinin kalıtsal bir torsiyon teorisine göre cebirsel özellikleri araştırılmıştır. $\mathrm{Bu}$ bağlamda özellikle, $\tau_{e^{-}}$ tümlenmiş ile bol $\tau_{e}$-tümlenmiş modüller arasındaki ilişkiler ve halka karakterizasyonları verilmiştir. $\quad \tau_{e^{-}}$ yükseltilebilir bir modülün her direkt toplam terimi de $\tau_{e}$-yükseltilebilirdir. Buna karş1lık, $\tau_{e}$-yükseltilebilir iki modülün de $\tau_{e^{-}}$ yükseltilebilir olmasını sağlayacak ön koşullar belirlenmiştir. $\tau_{e}$-tümlenmiş ve bol $\tau_{e}$-tümlenmiş modüllerin belli koşullar altında $\quad \tau_{e}$-yükseltilebilir olduğu ispatlanmıştır.

\section{Materyal ve Metot}

Tanım 2.1: $R$-mod da bir $\tau$-torsiyon teorisi aşağıdaki koşulları gerçekleyen $\tau=(\mathcal{T}, \mathcal{F})$ sınıflarının ikilisidir.

1. $\mathcal{T} \cap \mathcal{F}=\{0\}$.

2. $T \rightarrow M \rightarrow 0$ dizisi tam ve $T \in \mathcal{T}$ ise $M \in \mathcal{T}$ dir.

3. $0 \rightarrow M \rightarrow F$ dizisi tam ve $F \in \mathcal{F}$ ise $M \in \mathcal{F}$ dir.

4. Her $M$ modülü için $0 \rightarrow T \rightarrow M \rightarrow$ $F \rightarrow 0$ dizisi tam olacak şekilde $T \in$ $\mathcal{T}$ ve $F \in \mathcal{F}$ modülleri vardır (Bland, 1998).

Tanım 2.2: $M \in \mathcal{T}$ ise $M$ ye $\tau$-torsiyon modül; $M \in \mathcal{F}$ ise $M$ ye $\tau$-serbest torsiyon modül denir (Bland, 1998).

Tanım 2.3: $\tau=(\mathcal{T}, \mathcal{F}) \tau$-torsiyon teorisinde $\tau(M)=\sum\{N \leq M \mid N \in \tau\}$ olmak üzere
$\mathcal{T}=\{M \in R-\bmod \mid \tau(M)=M\} ; \quad \tau-$ torsiyon modüllerin sinıfıdır.

$\mathcal{F}=\{M \in R-\bmod \mid \tau(M)=0\} ; \quad \tau$ serbest torsiyon modüllerin sinıfidır (Bland, 1998).

Tanım 2.4: $\mathcal{T}$ sınıfı alt modüller altında kapalı ise yani içerdiği $\tau$-torsiyon modüllerin her alt modülü de $\tau$-torsiyon ise $\tau$ ya kalıtsal torsiyon teorisi denir (Bland, 1998).

Tanım 2.5: $\mathcal{F}$ sınıfı homomorfik görüntüler altında kapalı ise $\tau$ ya eşkalıtsal torsiyon teorisi denir (Bland, 1998).

Önerme 2.6: $\mathcal{T}$ sınıfi homomorfik görüntüler, direkt toplamlar ve genişlemeler altında kapalıdır (Bland, 1998).

Önerme 2.7: $\mathcal{F}$ sinıfı alt modüller, direkt çarpımlar ve genişlemeler altında kapalıdır (Bland, 1998).

Tanım 2.8: $M$ bir modül ve $N \leq M$ olsun. $M /{ }_{N}$ bölüm modülü $\tau$-torsiyon ise $N$ ye $M$ nin $\tau$-yoğun alt modülü denir ve " $N \leq_{\tau-d} M "$ ile gösterilir (Bland, 1998).

Tanım 2.9: $M$ bir modül ve $N \leq M$ olsun. $N \unlhd M$ ve $N \leq_{\tau-d} M$ ise $N$ ye $M$ nin $\tau$-büyük alt modülü denir ve " $N \unlhd_{\tau} M^{\prime \prime}$ ile gösterilir (Bland, 1998).

Her $\tau$-büyük alt modülün büyük alt modül olduğu açıktır. Ve tanımları gereği $\tau$-büyük alt modülün büyük alt modülün bir genelleştirilmiş versiyonu değil özelleştirilmişi olduğuna ulaşılabilir.

$\mathrm{Bu}$ bölümde verilen tüm tanım ve diğer bilgilerin detaylı formu için Bland (1998) a ait kaynak incelenebilir.

Tanım 2.10: $M$ bir modül ve $N \leq M$ olsun. $K \unlhd_{\tau} M$ için $N+K=M$ ifadesi yalnızca 
$K=M$ olduğunda gerçekleniyorsa $N$ ye $M$ nin $\tau_{e}$-küçük alt modül denir ve ${ }^{\prime \prime} N \ll_{\tau_{e}} M^{\prime \prime}$ ile gösterilir (Sözen, 2020).

Bir modülün her $e$-küçük alt modülü $\tau_{e^{-}}$ küçüktür. $\tau$-torsiyon modüller için tersi de söylenebilir.

Şimdi $\tau_{e}$-küçük alt modüllerin özelliklerini aşağıdaki önerme ile verelim.

Önerme 2.11: $M$ bir modül olsun.

1. $K, L, N \leq M$ ve $\mathrm{K} \leq N$ olsun.

a) $N \ll_{\tau_{e}} M$ ise $K \ll_{\tau_{e}} M$ ve $N / K \ll_{\tau_{e}} M / K$ dir.

b) $N+L \ll_{\tau_{e}} M \Leftrightarrow N \ll_{\tau_{e}} M$ ve $L \ll_{\tau_{e}} M$ dir.

2. $\tau_{e}$-küçük alt modüllerin homomorfik görüntüsü de $\tau_{e}$-küçük alt modüldür.

3. $K_{1} \leq M_{1} \leq M, K_{2} \leq M_{2} \leq M \quad$ ve $M=M_{1} \oplus M_{2} \quad$ olsun. $\mathrm{Bu}$ durumda, $K_{1} \oplus K_{2} \ll_{\tau_{e}} M_{1} \oplus M_{2} \Leftrightarrow K_{1} \ll_{\tau_{e}} M_{1} \quad$ ve $K_{2} \ll_{\tau_{e}} M_{2}$ dir.

4. $K \leq N \leq M, K \ll_{\tau_{e}} M$ ve $N, M$ nin direkt toplam terimi ise $K \ll_{\tau_{e}} N \operatorname{dir}$ (Sözen, 2020).

Tanım 2.12: $M$ bir modül ve $U, V \leq M$ olsun. $M=U+V$ ve bir $T \unlhd_{\tau} V$ için $M=U+T$ olması $\quad T=V$ olmasinı gerektiriyorsa $V$ ye $U$ nun $M$ de $\tau_{e}$-tümleyeni denir. Eğer $M$ nin her alt modülü $\tau_{e}$-tümleyene sahipse $M$ ye $\tau_{e}$ tümlenmiş modül denir (Sözen, 2020).

$E$-tümlenmiş her modül $\tau_{e}$-tümlenmiştir.

Lemma 2.13: $M$ bir modül ve $U, V \leq M$ olsun. $U$ alt modülünün $M$ de bir $V \tau_{e^{-}}$ tümleyene sahip olması için gerek ve yeter koşul $U+V=M$ ve $U \cap V \ll_{\tau_{e}} V$ olmasıdır (Sözen, 2020).

\section{Bulgular}

\section{Bol $\tau_{e}$-Tümlenmiş Modüller}

Tanım 3.1: $M$ bir modül ve $X \leq M$ olsun. Ĕger $M=X+K$ koşulunu gerçekleyen her $K$ alt modülü $X$ in $M$ de bir $\tau_{e}$-tümleyenini içeriyorsa bu takdirde $X, M$ de bol $\tau_{e^{-}}$ tümleyene sahiptir denir. Eğer $M$ nin her alt modülü $M$ de bir bol $\tau_{e}$-tümleyenine sahipse $M$ ye bol $\tau_{e}$-tümlenmiş modül adı verilir.

$\tau_{e}$-tümlenmiş ve bol $e$-tümlenmiş modüllerin bol $\tau_{e}$-tümlenmiş oldukları açıktır.

Teorem 3.2: $M$ bir modül ve $X$ ile $Y, M$ de bol $\tau_{e}$-tümleyene sahip ve $M=X+Y$ koşulunu gerçekleyen alt modüller olsun. $\mathrm{Bu}$ takdirde $X \cap Y$ alt modülü de $M$ de bol $\tau_{e^{-}}$ tümleyene sahiptir.

İspat: $W \leq M$ için $M=(X \cap Y)+W$ olsun. Modüler kuralı gereği, $X=(X \cap Y)+(W \cap$ $X)$ ve $Y=(X \cap Y)+(W \cap Y)$ elde edilir. Buradan, $M=X+Y$ olduğu için $M=X+$ $(W \cap Y)$ ve $M=Y+(W \cap X)$ yazilabilir. Hipotez gereği, $X$ ve $Y$ sirasiyla $M$ de $V_{1} \leq$ $W \cap Y$ ve $V_{2} \leq W \cap X$ olacak şekilde $V_{1}$ ve $V_{2} \tau_{e}$-tümleyenlerine sahiptir. Yani,

$X+V_{1}=M, X \cap V_{1} \ll_{\tau_{e}} V_{1}$ ve $Y+V_{2}=M$, $Y \cap V_{2} \ll_{\tau_{e}} V_{2}$

dir. Böylelikle $V_{1} \leq W \cap Y \leq Y$ için Modüler kuralı gereği $Y=V_{1}+(X \cap Y)$ dir. Benzer şekilde $X=V_{2}+(X \cap Y)$ olduğu görülebilir. Buradan $M=X+Y=(X \cap Y)+\left(V_{1}+V_{2}\right)$ elde edilir. Ayrıca

$$
\begin{aligned}
(X \cap Y) \cap\left(V_{1}\right. & \left.+V_{2}\right)=X \cap\left[Y \cap\left(V_{1}+V_{2}\right)\right] \\
& =X \cap\left[V_{1}+\left(V_{2} \cap Y\right)\right] \\
& =\left(X \cap V_{1}\right) \\
& +\left(V_{2} \cap Y\right) \ll_{\tau_{e}} V_{1}+V_{2}
\end{aligned}
$$

olduğundan $V_{1}+V_{2}, \quad X \cap Y \operatorname{nin} M$ de $V_{1}+$ $V_{2} \leq W$ olacak şekilde bir $\tau_{e}$-tümleyeni olur.

Teorem 3.3: Bol $\tau_{e}$-tümlenmiş bir modülün her bölüm modülü de bol $\tau_{e}$-tümlenmiştir. 
İspat: Standart şekilde yapılabilir.

Sonuç 3.4: Bol $\tau_{e}$-tümlenmiş bir modülün homomorfik görüntüsü de bol $\tau_{e^{-}}$ tümlenmiştir.

Teorem 3.5: Bir $M$ modülünün her alt modülü

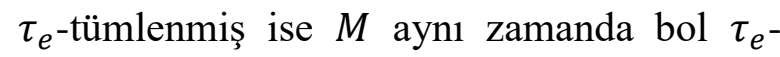
tümlenmiş modüldür.

İspat: $N \leq M$ ve bir $T \leq M$ için $M=N+T$ olsun. Hipotez gereği, $N \cap T, T$ de bir $K \tau_{e^{-}}$ tümleyenine sahiptir. Bu durumda $(N \cap T)+$ $K=T$ ve $(N \cap T) \cap K=N \cap K \ll_{\tau_{e}} K$ dir. Buradan $M=N+T=N+(N \cap T)+K=$ $N+K$ elde edilir. Sonuç olarak, $K, N \operatorname{nin} M$ de $T$ tarafindan kapsanan $\tau_{e}$-tümleyenidir dolayısıyla $M$ bol $\tau_{e}$-tümlenmiştir.

Hatıllatmak gerekirse, bir $M$ modülünün $X, Y \leq M$ olmak üzere $M=X+Y$ koşulunu gerçekleyen her $X, Y \leq M$ alt modülü için $f(M) \subseteq X$ ve $(I-f)(M) \subseteq Y$ olacak şekilde bir $f \in \operatorname{End}(M)$ var ise $M$ ye $\pi$-projektif modül denir. Her projektif modül $\pi$ projektiftir.

Teorem 3.6: $M$ modülü $\pi$-projektif ve $\tau_{e^{-}}$ tümlenmiş bir modül olsun. Bu takdirde $M$ bol $\tau_{e^{-} \text {tümlenmiş̧irir. }}$

İspat: $X, M$ nin herhangi bir alt modülü ve $Y \leq M$ için $M=X+Y$ olsun. $M$, $\pi$-projektif olduğundan $f(M) \subseteq Y$ ve $(I-f)(M) \subseteq X$ olacak şekilde bir $f \in \operatorname{End}(M)$ vardır. Ayrıca hipotez gereği $X, M$ de bir $T \tau_{e}$-tümleyenine sahiptir. Yani, $X+T=M$ ve $X \cap T \ll_{\tau_{e}} T$ dir. Buradan $M=f(M)+(I-f)(M)=$ $f(X)+f(T)+X=X+f(T)$ elde edilir. Ayrica $X \cap f(T) \leq f(X \cap T) \ll_{\tau_{e}} f(T)$ olup $f(T), M \operatorname{de} X$ in $Y$ de içerilen $\tau_{e}$-tümleyenidir.

Sonuç 3.7: $M$ modülü projektif ve $\tau_{e^{-}}$ tümlenmiş bir modül olsun. Bu takdirde $M$ bol $\tau_{e^{-} \text {-tümlenmiştir. }}$

Not: $\tau_{e}$-tümlenmiş modüllerin sonlu toplamları ve homomorfik görüntüleri de $\tau_{e^{-}}$ tümlenmiş olduğundan $\tau_{e}$-tümlenmiş bir $M$ modülünün her sonlu $M$-üretilmiş modülü de

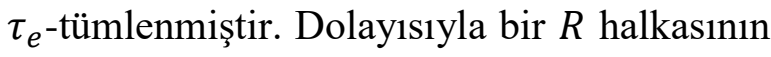
$\tau_{e}$-tümlenmiş olması için gerek ve yeter koşul her sonlu $R$-üretilmiş modülünün $\tau_{e^{-}}$ tümlenmiş olmasıdır.

Teorem 3.8: Bir $R$ halkası için aşağıdaki ifadeler denktir:

1. ${ }_{R} R$ modülü $\tau_{e}$-tümlenmiştir.

2. ${ }_{R} R$ modülü bol $\tau_{e}$-tümlenmiştir.

3. Her sonlu üretilmiş $R$-modül $\tau_{e^{-}}$ tümlenmiş̧ir.

4. Her sonlu üretilmiş $R$-modül bol $\tau_{e}$-tümlenmiş̧ir.

İspat: $(1 \Leftrightarrow 2)$ : Sonuç 3.7 gereği açıktır.

$(1 \Rightarrow 3)$ : Not 3.8 den açıktır.

$(3 \Rightarrow 4): M$ sonlu üretilmiş $\tau_{e}$-tümlenmiş bir modül olsun. Bu takdirde sonlu bir $I$ indeks kümesi için $f: R^{(I)} \rightarrow M$ epimorfizması mevcuttur. Hipotez gereği $R^{(I)} \tau_{e}$-tümlenmiş olup $R$ projektif olduğundan

$R^{(I)}$ (sonlu kopyalarının toplamı) projektiftir. Not 3.8 gereği $R^{(I)}$ bol $\tau_{e}$-tümlenmiştir.

$(4 \Rightarrow 1)$ : Açıktır.

\section{$\tau_{e}-$ Yükseltilebilir Modüller}

Tanım 3.9: $M$ bir modül ve $N \leq M$ olsun. Bu durumda $M$ nin $X \leq N$ ve $N \cap Y \ll_{\tau_{e}} M$ olacak şekilde bir $M=X \oplus Y$ ayrışımı mevcut ise $M$ ye $\tau_{e}$-yükseltilebilir modül denir.

Her $\quad e$-yükseltilebilir modül $\tau_{e^{-}}$ yükseltilebilirdir. Ayrica $\tau$-torsiyon serbest her $\tau_{e}$-yükseltilebilir modül ise $e$ yükseltilebilirdir. Bununla birlikte $\tau_{e^{-}}$ yükseltilebilir modüller $\tau_{e}$-tümlenmiştir.

Aşağıldaki örnekte $\tau_{e}$-tümlenmiş bir modülün $\tau_{e}$-yükseltilebilir olmayabileceği 
gösterilmektedir.

Örnek 3.10: $p$ bir asal tam say1, $R=\mathbb{Z}_{p^{3}}$ ve $M=\mathbb{Z} / p^{3} \mathbb{Z} \oplus \mathbb{Z} / p \mathbb{Z} \quad$ olsun. $\quad R \quad$ halkasi mükemmel olduğundan ${ }_{R} R$ modülü $\tau_{e^{-}}$ tümlenmiştir ancak $\tau_{e}$-yükseltilebilir değildir.

Aşağıda bir modülün $\tau_{e}$-yükseltilebilir olmasına eşdeğer koşullar sıralanmaktadır.

\section{Lemma 3.11:}

1. Bir $M$ modülü için aşağıdaki ifadeler denktir:

a) $M \tau_{e}$-yükseltilebilirdir.

b) $M$ nin herhangi bir $N$ alt modülü için $X, M$ nin bir direkt toplam terimi ve $Y \ll_{\tau_{e}} M$ olacak şekilde $N=X \oplus Y$ parçalanışı mevcuttur.

c) $M$ nin herhangi bir $N$ alt modülü için $X \leq N \quad$ ve $N / X \ll_{\tau_{e}} M / X$ olacak şekilde $M$ de bir $X$ direkt toplam terimi mevcuttur.

2. $\tau_{e}$-yükseltilebilir bir modülün her direkt toplam terimi de $\tau_{e^{-}}$ yükseltilebilirdir.

İspat: 1 . $(1 a \Rightarrow 1 b)$ : Açıktır.

$(1 b \Rightarrow 1 c): N \leq M$ olsun. Hipotez gereği $N$ nin, $M$ nin bir direkt toplam terimi ve $Y \ll_{\tau_{e}} M \quad$ olacak şekilde $\quad N=X \oplus Y$ parçalanış1 mevcuttur. $\pi: M \rightarrow M / X$ doğal homomorfizması için $Y \ll_{\tau_{e}} M$ olduğundan $\pi(Y)=Y+X /{ }_{X}=N /{ }_{X} \ll_{\tau_{e}} M / X$ dir.

$(1 c \Rightarrow 1 a): N \leq M$ olsun. Hipotez gereği $M$ nin, $X \leq N$ ve $N / X \ll_{\tau_{e}} M / X$ olacak şekilde $M=X \oplus Y \quad$ parçalanışı mevcuttur. Dolayısıyla, açıkça $M=N+Y$ ve $N=X \bigoplus$ $(Y \cap N)$ elde edilir. Ayrıca $M / X \cong Y$ ve $N / X \cong Y \cap N$ olduğundan $Y \cap N \ll_{\tau_{e}} Y$ olur.
Sonuç olarak $M \tau_{e}$-yükseltilebilirdir.

2. $\quad M \tau_{e}$-yükseltilebilir bir modül ve $N \leq$ $M$ bir direkt toplam terimi olsun. Bu takdirde bir $T \leq M$ için $M=N \oplus T$ dir. $X \leq N \leq M$ için $M \tau_{e}$-yükseltilebilir olduğundan $Z \leq X$ ve $X \cap Y \ll_{\tau_{e}} Y$ olmak üzere $M=Z \oplus Y$ parçalanışı mevcuttur. Dolayısıyla $N=Z \oplus$ $(N \cap Y) ; N \cap(X \cap Y)=X \cap(N \cap Y)=X \cap$ $Y \ll_{\tau_{e}} N \cap Y$.

Lemma 3.11 de $\tau_{e}$-yükseltilebilir modüllerin sınıfinin direkt toplamlar altında kapalı olduğu ispatlandı. Aşağıdaki örnekte $\tau_{e^{-}}$ yükseltilebilir modüllerin sınıfının bölüm modülleri için kapalı olmadığ gösterilmektedir. Ardından belli koşullar altında bunun doğrulandığını gösteren önerme verilecektir.

Örnek 3.12: $p$ bir asal tam sayı, $R=\mathbb{Z}_{p^{3}}$ ve $M=\mathbb{Z}_{p^{3}} \oplus\left({ }^{p \mathbb{Z}} / p^{2} \mathbb{Z}\right)$ olsun. $M,\left(R^{2}\right)$ nin bir homomorfik görüntüsü olup $R$ halkası mükemmel olduğundan $\left(R^{2}\right)$ serbest modülü de $\tau_{e}$-yükseltilebilirdir. Diğer taraftan, Örnek 3.1 gereği $M, \tau_{e}$-yükseltilebilir değildir.

$\forall X, Y, Z \leq M$ için $X \cap(Y+Z)=(X \cap Y)+$ $(X \cap Z)$ oluyorsa $M$ ye dağılımlı modül adı verilir. Ayrıca $\forall f \in \operatorname{End}(M)$ için $f(X) \subseteq X$ oluyorsa $X \leq M$ alt modülüne tam invaryanttır denir.

Önerme 3.13: $M \tau_{e}$-yükseltilebilir bir modül ve $X \leq M$ olsun. Aşağıdaki koşullardan biri sağlandığı takdirde $M / X$ bölüm modülü de $\tau_{e^{-}}$ yükseltilebilirdir.

1. $\quad N, M$ nin direkt toplam terimi olmak üzere $N+X /{ }_{X}, M / X$ in direkt toplamidir.

2. $\quad M$ dağglımlı modüldür.

3. $f=f^{2} \in \operatorname{End}(M)$ için $f(X) \subseteq X$ dir. 
Özel olarak $X, M$ nin tam invaryant alt modülüdür.

\section{İspat:}

1. $K / X \leq M / X$ olsun. $K \leq M$ ve $M \tau_{e^{-}}$ yükseltilebilir olduğundan $T \leq K$ ve $K / T \ll \tau_{e} M / T$ olacak şekilde $M$ de bir $T$ direkt toplam terimi mevcuttur. Burada $T+X / X \leq M / X$ bir direkt toplam terimi ve $T+X / X \leq K / X \leq M / X$ dir. $K / /_{T} \ll_{\tau_{e}} M / T$ olduğundan $K / T+X \ll_{\tau_{e}} M / T+X \quad$ dir. Sonuç olarak $M / X$ bölüm modülü de $\tau_{e^{-}}$ yükseltilebilirdir.

2. Birinci koşuldan faydalanarak ispat yapılacaktır. $M=Y \oplus Z$ olsun. Bu durumda $M / X=Y+X /{ }_{X}+Z+X /{ }_{X}$ ve $(Y+X / X) \cap$ $\left(Z+X /{ }_{X}\right)=(Y \cap Z)+X /{ }_{X}=0{ }_{M} \quad$ dir. Sonuç olarak $Y+X /{ }_{X}, M / X$ in direkt toplam terimi olup $M /{ }_{X} \tau_{e}$-yükseltilebilirdir.

3. $M=A \oplus B$ olsun. Birinci koşul gereği $A+X / X, M / X$ in direkt toplam terimi olduğu gösterilmelidir. $\pi: A \oplus B \rightarrow A,(1-\pi) M=$ $B$ çekirdeğine sahip projeksiyon dönüşümü olsun. $\mathrm{Bu}$ takdirde $\pi^{2}=\pi \in \operatorname{End}(M)$ ve $\pi(M)=A$ dır. Hipotez gereği, $\pi(X) \leq X$ ve $(1-\pi) X \leq X$ dir. Bu durumda $\pi(X)=X \cap$ $A$ ve $(1-\pi) X=X \cap B$ dir. Dolayısıyla $X=$ $\pi(X) \oplus(1-\pi)(X)=(X \cap A) \oplus(X \cap B)$ dir. Buradan $A+X / X=A \oplus(X \cap B) /{ }_{X}$ ve $B+X / X=B \oplus(X \cap A) / X$ olduğu kolayca görülür. Böylece $M / X_{X}=A \oplus(X \cap B) / X^{+}$ $B \oplus(X \cap A) /_{X}$ elde edilir. Ayrıca Modüler kuralından $\quad[A \oplus(X \cap B)] \cap[B \oplus(X \cap$ $A)]=[A \oplus(X \cap B)] \cap B \oplus(X \cap A)=$ $(X \cap B) \oplus(A \cap B) \oplus(X \cap A)=X \cap B \oplus$ $X \cap A=X$ olup $A+X / X, M / X$ in direkt toplam terimidir.
Lemma 3.11 de $\tau_{e}$-yükseltilebilir bir modülün her direkt toplam teriminin de $\tau_{e^{-}}$ yükseltilebilir olduğu gösterildi. Fakat tersinin doğrulandığını söylemek genelde mümkün değildir. Teorem 3.15 ile uygun koşullar altında bunun sağlandığ1 gösterilecektir. Ancak öncesinde aşağıdaki lemmaya ihtiyaç vardır.

Lemma 3.14: $M=X \oplus Y$ olsun. Bu takdirde aşağıdaki ifadeler denktir:

1. $X, Y$-projektiftir.

2. Her $T \leq M$ için $M=T+Y$ ise $M=$ $T^{\prime} \oplus Y$ olacak şekilde bir $T^{\prime} \leq T$ vardır (Wisbauer, 41.14).

Teorem 3.15: $X, M$ nin self-projektif ve $Y$ projektif bir alt modülü olmak üzere $M=$ $X \oplus Y$ olsun. $X$ ve $Y, M$ nin $\tau_{e}$-yükseltilebilir alt modülleri ise $M$ de $\tau_{e}$-yükseltilebilirdir.

İspat: $N \leq M$ olsun. $X \cap(N+Y) \leq X$ için $X$ $\tau_{e}$-yükseltilebilir olduğundan $D \leq X \cap(N+$ $Y)$ ve $\left(X \cap(N+Y) \cap D^{\prime}=(N+Y) \cap\right.$ $D^{\prime} \ll_{\tau_{e}} D^{\prime}$. Böylece $M=X \oplus Y=D \oplus$ $D^{\prime} \oplus Y=N+D^{\prime} \oplus Y$ dir. $X$ self ve $Y$ projektif olduğundan aynı zamanda $M$ projektiftir. $D \rightarrow D \oplus\left(D^{\prime} \oplus Y\right) \rightarrow D^{\prime} \oplus Y$ tam dizisi göz önüne alınarak $D \operatorname{nin} D^{\prime} \oplus Y$ projektif olduğu söylenebilir. Dolayısıyla Lemma 3.14 gereği $M=N^{\prime} \oplus\left(D^{\prime} \oplus Y\right)$ olacak şekilde bir $N^{\prime} \leq N$ vardır. Buna göre $W \leq Y$ için $N \cap\left(W+D^{\prime}\right)=W \cap\left(N+D^{\prime}\right)$ yazılabilir. Diğger taraftan, $Y \tau_{e}$-yükseltilebilir olduğundan $Y_{1} \leq N \cap\left(Y+D^{\prime}\right)=Y \cap(N+$ $\left.D^{\prime}\right)$ ve $Y_{2} \cap\left(N+D^{\prime}\right) \ll_{\tau_{e}} Y_{2}$ olacak şekilde $Y=Y_{1} \oplus Y_{2}$ ayrışımı mevcuttur. Dolayısıyla $M=N^{\prime}+\left(Y+D^{\prime}\right)=N^{\prime} \oplus\left(D^{\prime} \oplus Y_{1} \oplus\right.$ $\left.Y_{2}\right)=\left(N^{\prime} \oplus Y_{1}\right) \oplus\left(Y_{2} \oplus D^{\prime}\right) \quad$ olduğu kolayca görülebilir. $N^{\prime} \leq N$ ve $X \leq N \cap(Y+$ $\left.D^{\prime}\right) \leq N$ olduğundan $N^{\prime} \oplus Y_{1} \leq N$ olur ve dolayısıyla $M=N+\left(Y \oplus D^{\prime}\right)$ olur. Ayrica $N \cap\left(Y_{2} \oplus D^{\prime}\right)=Y_{2} \cap\left(N+D^{\prime}\right) \ll_{\tau_{e}} Y_{2} \leq$ $Y+D^{\prime}$ dir. 
Sonuç 3.16: $X$ ve $Y, X$-projektif modüller olmak üzere $X$ yarı basit ve $Y, \tau_{e^{-}}$ yükseltilebilir olsun. Bu takdirde $M=X \oplus Y$ modülü de $\tau_{e}$-yükseltilebilirdir.

Örnek 3.17: $M=\mathbb{Z}_{3} \oplus \mathbb{Z}_{27}, \quad \mathbb{Z}$-modülünün her bir direkt toplam terimi oyuk olduğundan $\tau_{e}$-yükseltilebilirdir. Ancak $\mathbb{Z}_{3}$ ün $\mathbb{Z}_{27^{-}}$ projektif olmadığ göz önüne alınırsa $M$ nin kendisi $\tau_{e}$-yükseltilebilir değildir.

Aşağıdaki teoremde duo modüller (alt modülleri tam invaryant olan modüller) için $\tau_{e}$-yükseltilebilir iki modülün direkt toplamının da $\tau_{e}$-yükseltilebilir olduğu gösterilmektedir.

Önerme 3.18: $M=X \bigoplus Y$ bir duo modül olsun. $X$ ve $Y, \tau_{e}$-yükseltilebilir modüller ise $M$ de $\tau_{e}$-yükseltilebilirdir.

İspat: $N \leq M$ olsun. $M$ bir duo modül olduğundan $\quad N=(N \cap X) \oplus(N \cap Y)$ şeklimde yazılabilir. Hipotez gereği $N \cap X \leq$ $X, N \cap Y \leq Y$ alt modülleri için $X=X_{1} \oplus$ $X_{2}, \quad X_{1} \leq N \cap X, \quad N \cap X_{2} \ll_{\tau_{e}} X_{2}$ ve $Y=$ $Y_{1} \oplus Y_{2}, Y_{1} \leq N \cap Y, N \cap Y_{2} \ll_{\tau_{e}} Y_{2}$ olacak şekilde $X_{1}, X_{2} \leq X$ ve $Y_{1}, Y_{2} \leq Y$ alt modülleri mevcuttur. Dolayisiyla, $\quad M=X \oplus Y=$ $\left(X_{1} \oplus X_{2}\right) \oplus\left(Y_{1} \oplus Y_{2}\right)=\left(X_{1} \oplus Y_{1}\right) \oplus$ $\left(X_{2} \oplus Y_{2}\right)$ dir. Buradan, $X_{1} \oplus Y_{1} \leq(N \cap$ $X) \oplus(N \cap Y)=N \cap(X \oplus Y)=N \cap M=$ $M \quad$ ve $N \cap\left(X_{2} \oplus Y_{2}\right)=\left(N \cap X_{2}\right) \oplus(N \cap$ $\left.Y_{2}\right) \ll_{\tau_{e}} X_{2} \oplus Y_{2}$ dir.

Aşağıdaki önermelerde $\tau_{e}$-tümlenmiş ve bol $\tau_{e}$-tümlenmiş modüllerin $\tau_{e}$-yükseltilebilir olma koşulları verilmektedir.

Önerme 3.19: $M$ her $\tau_{e}$-tümleyen alt modülü bir direkt toplam terimi olan projektif $\tau_{e^{-}}$ tümlenmiş bir modül olsun. Bu takdirde $M \tau_{e^{-}}$ yükseltilebilirdir.

İspat: $X \leq M$ olsun. $M \tau_{e}$-tümlenmiş modül olduğundan bir $Y \leq M$ alt modülü $X+Y=M$ ve $X \cap Y \ll_{\tau_{e}} Y$ olacak şekilde mevcuttur. Bu durumda $M$ projektif ve $M=X+Y$ olduğundan Lemma 3.14 gereği $D \subseteq X$ dir. Sonuç olarak $M$ nin her $X$ alt modülü için $D \subseteq$ $X$ ve $X \cap Y \ll_{\tau_{e}} Y$ olacak şekilde. $M=Y \oplus$ $D$ parçalanışı bulunmuş olur. Bu takdirde $M$ modülü $\tau_{e}$-yükseltilebilirdir.

Önerme 3.20: $M$ her $\tau_{e}$-tümleyen alt modülü bir direkt toplam terimi olan bol $\tau_{e}$-tümlenmiş bir modül olsun. $\mathrm{Bu}$ takdirde $M \tau_{e^{-}}$ yükseltilebilirdir.

İspat: $\quad M$ bol $\tau_{e}$-tümlenmiş modül olduğundan $M$ nin herhangi bir $X$ alt modülü için bir $Y \quad \tau_{e}$-tümleyeni; ve $Y \leq M$ alt modülüne karşılık da $Y^{\prime} \leq M$ (bol) $\tau_{e^{-}}$ tümleyeni $\quad Y^{\prime} \subseteq X, M=Y^{\prime} \oplus D \quad$ olacak şekilde mevcuttur. $M=Y^{\prime} \oplus D$ olacak şekilde mevcuttur. $M=Y^{\prime} \oplus Y$ olup $X=$ $Y^{\prime}+(Y \cap X)=Y^{\prime} \oplus(X \cap D)$ elde edilir. $\pi: Y^{\prime} \oplus D \rightarrow D$ izdüşüm fonksiyonu olmak üzere $\pi(Y \cap X)=\pi(X)=X \cap D$ dir. Ayrıca $Y \cap X \ll_{\tau_{e}} Y$ olduğundan $\pi(Y \cap X)=X \cap$ $D \ll_{\tau_{e}} \pi(Y) \subseteq D$ olup $X \cap D \ll_{\tau_{e}} D$ dir. Böylelikle, $M$ nin her $X$ alt modülü için $Y^{\prime} \subseteq$ $X$ ve $X \cap D \ll_{\tau_{e}} D$ olacak şekilde $M$ nin $M=Y^{\prime} \oplus D$ parçalanışı mevcuttur.

\section{Teșekkür}

Makalenin değerlendirilmesine ve geliştirilmesine katkı sağlayan değerli hakemlere teşekkür ederiz.

\section{Kaynaklar}

Bland, P.E. (1998). "Topics in Torsion Theory", Mathematical Research, Wiley VCH Verlag Berlin GmbH, 103. Berlin.

Charalambides, S. 2006 "Topics in Torsion Theory", PhD Thesis, University of Otago, Dunedin, New Zealand. 
Clark, J., Lomp, C., Vanaja, N. and Wisbauer, R. (2006). "Lifting Modules", Birkhauser, Basel.

Koşan, T. and Harmanc1, A. 2004. "Modules Supplemented with Respect to a Torsion Theory", Turkish Journal of Mathematics, 28: 177-184.

Koşar, B., Nebiyev, C. and Sökmez, N. 2015. "G-supplemented Modules", Ukrainian Mathematical Journal, 67: 861-864.

Quynh, T.C. and Tin, P.H. 2013. "Some Properties of $e$-Supplemented and $e$-Lifting Modules", Vietnam Journal of Mathematics", 41: 303-312.

Sözen, Ö.E. 2020. “E-tümlenmiş Modüllerin Torsiyon Teorisine Göre Genelleştirilmiş Versiyonu", 13 (Özel Say1 I), 76-82, (32. Ulusal Matematik Sempozyumu, Ondokuz Mayıs Üniversitesi, Samsun).

Zhou, D.X., Zhang, X.R., 2011. "Smallessential Submodules and Morita Duality", Southeast Asian Bull. Math., 35: 1051-1062. 